\title{
乳酸催化的酰胺与胺的氨解反应
}

\author{
刘巨艳* 赵聪颖 \\ (天津师范大学化学学院 天津市功能分子结构与性能重点实验室 天津 300387)
}

\begin{abstract}
摘要 发展了一种对环境友好的乳酸促进的酰胺与胺的氨解的新方法, 该方法能够成功合成一系列芳香、脂肪类酰胺 和腿的衍生物. 该方法最显著的特征是催化剂廉价易得、无挥发性溶剂、底物转化率高及产物提纯操作简单.

关键词 乳酸; 酰胺; 腿; 氨解
\end{abstract}

\section{Lactic Acid-Catalyzed Transamidation Reactions of Carboxamides with Amines}

\author{
Liu, Juyan* Zhao, Congying \\ (Tianjin Key Laboratory of Structure and Performance for Functional Molecules, College of Chemistry, \\ Tianjin Normal University, Tianjin 300387)
}

\begin{abstract}
An environmentally benign protocol for the transamidation of carboxamides with amines using lactic acid as a green catalyst has been developed. The method has been successfully applied to the synthesis of a wide range of aromatic and aliphatic amides and ureas. The reaction has the advantages of the ready accessibility of the catalyst, solvent-free condition, efficient transformation and green processing.

Keywords lactic acid; carboxamide; urea; transamidation
\end{abstract}

酰胺键作为一类常见的化学键, 广泛存在于工业和 医药化学领域 ${ }^{[1]}$. 酰胺类化合物作为一类重要的有机化 合物，被广泛应用于生物活性产物、生物高分子材料(如 蛋白质)和工业高分子材料(如尼龙)的合成. 2007 年, 美 国化学会绿色化学机构药物圆桌会议(ACSGCIPR)表决 认为 “酰胺形成一避免低原子经济性试剂” 应该作为有 机化学的顶级目标 ${ }^{2]}$. 该会议还指出, 全球主要医药公 司生产的药物分子中 $25 \%$ 包含有酰胺单元，这说明了酰
胺结构在合成有机化学领域的重要性和普遍性. 图 1 为 国际上大型医药公司开发的具有酰胺键的药物分子 ${ }^{[3-5]}$.

首先，制备酰胺的传统方法是建立在活化后的羧酸 衍生物与胺的偶联基础之上 ${ }^{[6]}$. 但传统方法的总体缺陷 是需要化学计量的活化试剂、额外的分离步骤及繁琐的 操作过程. 其次, 通过催化着酸与胺缩合直接合成酰胺 反应的副产物只有水，这是更为绿色、简便的合成方 法 ${ }^{[7]}$. 近些年来，化学家发现酰胺的氨解是直接制备酰<smiles>CC(NC(=O)N(C)C1CCN(C)CC1)c1ccc(-c2cnccn2)c(Cl)c1Cl</smiles>

Helsinn, H0700, Ref. [3]

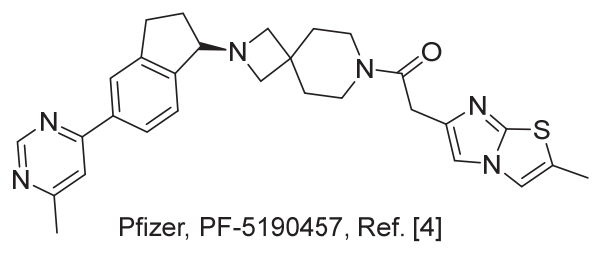

图 1 具有酰胺键药物分子实例

Figure 1 Examples of drug molecules containing amide bond

* Corresponding author. E-mail: hxxyljy@mail.tjnu.edu.cn

Received October 9, 2020; revised December 28, 2020; published online March 25, 2021.

Project supported by the Natural Science Foundation of Tianjin City (No. 12JCZDJC34300) and the College Students' Entrepreneurship Training Program (No. 201447).

天津市自然科学基金(No. 12JCZDJC34300)和大学生创业训练(No. 201447)资助项目. 
胺键非常有吸引力的方法, 文献中报道的主要金属催化 剂有 $\mathrm{MnCl}_{2} 、 \mathrm{TiCl}_{4} 、 \mathrm{AlCl}_{3}{ }^{[8]} 、 \mathrm{Sc}(\mathrm{OTf})_{3}$ 络合物、 $\mathrm{Al}_{2}\left(\mathrm{NMe}_{2}\right)_{6}$ 络合物、 $\mathrm{Ti}\left(\mathrm{NMe}_{2}\right)_{4}$ 络合物 ${ }^{[9-10]} 、 \mathrm{Cu}(\mathrm{OAc})_{2}{ }^{[11]} 、 \mathrm{KSF}$ polyDMAP 负载的 $\mathrm{HfCl}_{4}{ }^{[12]} 、 \mathrm{CeO}_{2}{ }^{[13]} 、 \mathrm{Fe}\left(\mathrm{NO}_{3}\right)_{3} \cdot 9 \mathrm{H}_{2} \mathrm{O}^{[14]}$ 、 磺化钨酸盐 ${ }^{[15]}$ 和 $\mathrm{Fe}^{3+}$-交换的蒙脱石 ${ }^{[16]}$; 文献中报道的

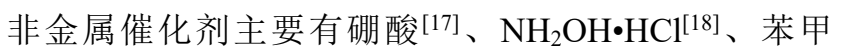

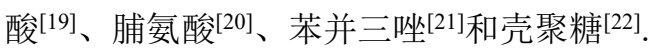

乳酸是广泛存在于自然界的天然有机物, 也是化工 界一种多功能生物基化学品，被广泛应用于食品、制药、 化妆品及化学品行业 ${ }^{[23-25]}$. 作为重要的基础原料乳酸可 被用于生产重要的化学品如丙烯酸、丙酮酸、丙二醇、 乙酰丙酮、2,3-戊二酮及乙醛 ${ }^{[23-24]}$. 此外, 乳酸还是生产 聚乳酸的单体, 而聚乳酸是一种生物可降解的塑料和石 油基聚合物的潜在替代品 ${ }^{[26-28]}$. 近年来, 化学家们逐渐 发现乳酸可以作为有机化学反应催化剂、溶剂使用, 表 现了低挥发性、高效率及绿色环保的特点 ${ }^{[29-30]}$. 在本课 题组 ${ }^{[31]}$ 开展的小分子催化合成研究工作的基础上, 本 工作采用乳酸作为催化剂, 在无溶剂条件下发展了酰 胺、䐖与胺之间的氨解反应, 在较短时间内以较高收率 合成了一系列酰胺化合物(Eq. 1).

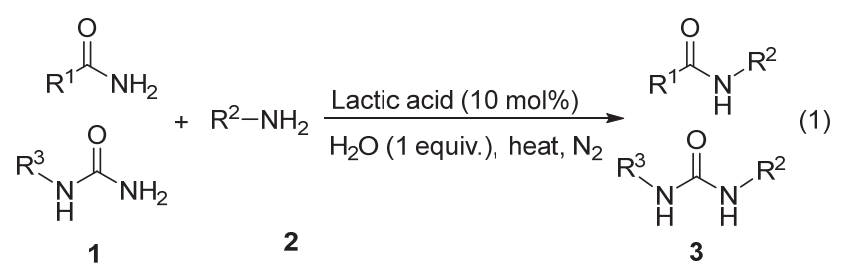

\section{1 结果与讨论}

\section{1 反应条件的优化}

为了确定最佳的反应条件, 在温度为 $40{ }^{\circ} \mathrm{C}$ 时, 以 甲酰胺 $(1.0 \mathrm{mmol})$ 和苄胺 $(1.0 \mathrm{mmol})$ 作为底物的反应为 模型反应，分别以柠檬酸(Citric acid)、苹果酸 $(D / L-M a l i c$ acid)、酒石酸 $(D / L$-Tartaric acid)、葡萄糖酸(Gluconic acid)、乳酸乙酯(Ethyl lactate)、乳酸 $[( \pm)$-Lactic acid]为 催化剂, 通过添加一定用量的水和有机溶剂, 进行了一 系列的实验，反应结果如表 1 所示. 不难发现，加入的 不同种类的催化剂对反应收率有着较大的影响(表 1 , Entries $2 \sim 7)$. 溶剂量的水和有机溶剂的加入以及室温 条件降低了产物的收率(表 1, Entries 8 12). 在无催化 剂的条件下，反应进行足够长的时间，却几乎没有预期 产物生成，这说明乳酸在反应过程中起到了十分重要的 作用(表 1, Entry 1). 在 $10 \mathrm{~mol} \%$ 乳酸为催化剂的条件下， 加入 1 equiv.的水, 反应效果最好(表 1, Entry 7), 过量的 催化剂并不能显著增加产物的收率(表 1, Entry 13). 在 只加入水而不加入任何催化剂的条件下，反应进行得并 不顺利, 底物的转化率极低(表 1, Entry 14). 因此, 最后 确定最佳反应条件为: $\mathrm{N}_{2}$ 保护下, $40{ }^{\circ} \mathrm{C}$, 无溶剂, 10 $\mathrm{mol} \%$ 乳酸为催化剂, 与乳酸等物质的量的水为添加剂.

\section{2 反应底物的普适性研究}

在上述最佳反应条件下, 考察了乳酸催化酰胺与胺 的交换反应体系的催化效率和适用范围. 将酰胺反应物

表 1 酰胺与胺之间氨解条件的优化 ${ }^{a}$

Table 1 Optimization of conditions for the transamidation of carboxamides with amines

\begin{tabular}{|c|c|c|c|c|}
\hline Entry & Catalyst/additive or solvent & Time/h & Temp. $/{ }^{\circ} \mathrm{C}$ & Yield $b / \%$ \\
\hline 1 & None & 24 & 40 & Trace \\
\hline 2 & Citric acid $(10 \mathrm{~mol} \%) / \mathrm{H}_{2} \mathrm{O}$ (1 equiv.) & 10 & 40 & 50 \\
\hline 3 & $D / L$-Malic acid $(10 \mathrm{~mol} \%) / \mathrm{H}_{2} \mathrm{O}$ (1 equiv.) & 10 & 40 & 53 \\
\hline 4 & $D / L$-Tartaric acid $(10 \mathrm{~mol} \%) / \mathrm{H}_{2} \mathrm{O}$ (1 equiv.) & 10 & 40 & 46 \\
\hline 5 & Gluconic acid (10 mol\%) & 10 & 40 & 64 \\
\hline 6 & Ethyl lactate $(10 \mathrm{~mol} \%) / \mathrm{H}_{2} \mathrm{O}$ (1 equiv.) & 10 & 40 & 76 \\
\hline 7 & $( \pm)$-Lactic acid $(10 \mathrm{~mol} \%) / \mathrm{H}_{2} \mathrm{O}$ (1 equiv.) & 10 & 40 & 95 \\
\hline 8 & $( \pm)$-Lactic acid $(10 \mathrm{~mol} \%) / \mathrm{H}_{2} \mathrm{O}(2 \mathrm{~mL})$ & 10 & 40 & 61 \\
\hline 9 & $( \pm)$-Lactic acid $(10 \mathrm{~mol} \%) /$ Toluene $(2 \mathrm{~mL})$ & 10 & 40 & 49 \\
\hline 10 & $( \pm)$-Lactic acid $(10 \mathrm{~mol} \%) / \mathrm{DMF}(2 \mathrm{~mL})$ & 10 & 40 & 56 \\
\hline 11 & $( \pm)$-Lactic acid $(10 \mathrm{~mol} \%) / \mathrm{DMSO}(2 \mathrm{~mL})$ & 10 & 40 & 59 \\
\hline 12 & $( \pm)$-Lactic acid $(10 \mathrm{~mol} \%) / \mathrm{H}_{2} \mathrm{O}$ (1 equiv.) & 10 & 25 & 30 \\
\hline 13 & $( \pm)$-Lactic acid $(20 \mathrm{~mol} \%) / \mathrm{H}_{2} \mathrm{O}$ (1 equiv.) & 10 & 40 & 90 \\
\hline 14 & $\mathrm{H}_{2} \mathrm{O}$ (1 equiv.) & 10 & 40 & $<5$ \\
\hline
\end{tabular}

${ }^{a}$ All reactions were carried out under nitrogen protection with formamide $(1.0 \mathrm{mmol})$, benzylamine $(1.0 \mathrm{mmol})$ and catalyst $(10 \mathrm{~mol} \%) .{ }^{b}$ Yield of isolated product. 
的范围扩大至芳香酰胺、脂肪酰胺、杂环酰胺和脲, 胺 反应物范围扩大至脂肪胺、芳香胺、一级胺、二级胺和 手性胺, 实验结果见表 2 . 从表 2 可以看出, 当酰胺底物 为甲酰胺时, 当另一种底物为苯胺衍生物时, 苯环上连 有供电子基的胺(表 2, Entries 1,3)比连有吸电子基的胺 (表 2, Entry 2)更容易进行反应，大部分反应在较短时间 内即可完成并能获得较高的收率. 链状脂肪一级胺与甲
酰胺、乙酰胺的反应活性(表 2, Entries 4, 9, 11)比二级 胺、环状脂肪一级胺的反应活性高很多(表 2, Entries 5 $8)$, 这可能是由于二级胺、环状脂肪一级胺的空间体积 大，使产品收率明显减少的缘故. 值得注意的是，具有 光学活性的胺类 $(S)-1$-苯乙胺在与甲酰胺反应生成产物 后，没有发生消旋现象(表 2, Entry 10).

表 2 乳酸催化酰胺与胺之间的交换反应 ${ }^{a}$

Table 2 Transamidation reactions of various amides and amines catalyzed by lactic acid

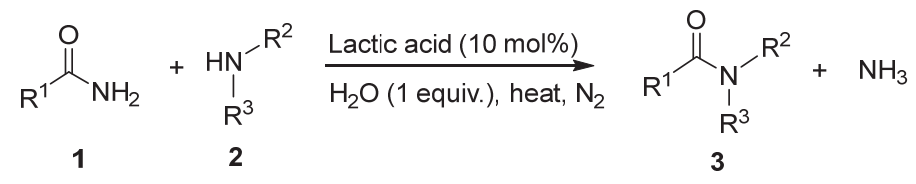

\begin{tabular}{|c|c|c|c|c|c|c|}
\hline Entry & Amide & Amine & Product & Time/h & Temp. $/{ }^{\circ} \mathrm{C}$ & Yield $^{b} / \%$ \\
\hline 1 & & & $3 a$ & 5 & 80 & 95 \\
\hline 2 & & & $3 b$ & 7 & 150 & 91 \\
\hline 3 & & & & 4 & 75 & 96 \\
\hline 4 & & & & 10 & 40 & 95 \\
\hline 5 & & & & 15 & 100 & 79 \\
\hline 6 & & & & 10 & 150 & 87 \\
\hline 7 & & & & 19 & 100 & 83 \\
\hline 8 & $\mathrm{H}_{2}$ & & & 19 & 100 & 84 \\
\hline 9 & $\mathrm{NH}_{2}$ & & & 19 & 100 & 91 \\
\hline 10 & & & & 12.5 & 40 & 82 \\
\hline
\end{tabular}




\begin{tabular}{|c|c|c|c|c|c|c|}
\hline Entry & Amide & Amine & Product & Time/h & Temp. $/{ }^{\circ} \mathrm{C}$ & Yield $^{b} / \%$ \\
\hline 11 & & & 3k & 8 & 110 & 97 \\
\hline 12 & & & 31 & 2 & 140 & 95 \\
\hline 13 & & & $3 m$ & 10 & 110 & 81 \\
\hline 14 & & & $3 n$ & 6 & 140 & 89 \\
\hline 15 & & & 30 & 14 & 140 & 88 \\
\hline 16 & & & & 16 & 140 & 87 \\
\hline 17 & & & & 20 & 150 & 82 \\
\hline 18 & & & $3 r$ & 14 & 120 & 90 \\
\hline 19 & & & & 13.5 & 140 & 94 \\
\hline 20 & & & & 11 & 130 & 97 \\
\hline 21 & & & & 6 & 140 & 88 \\
\hline 22 & & & & 10 & 140 & 82 \\
\hline
\end{tabular}




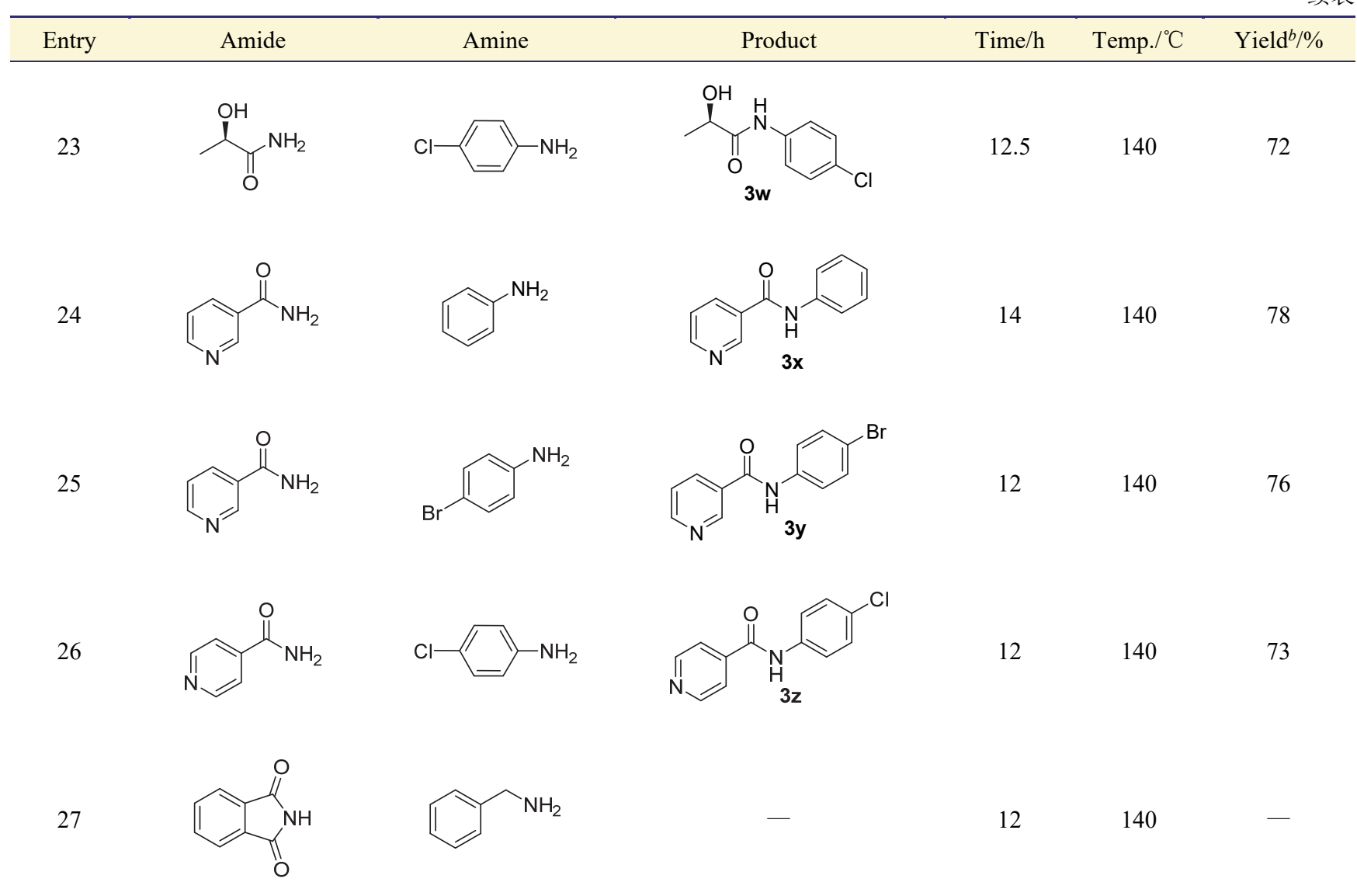

${ }^{a}$ Reaction conditions: amide $(1.0 \mathrm{mmol})$, amine $(1.0 \mathrm{mmol})$, lactic acid $(10 \mathrm{~mol} \%) / \mathrm{H}_{2} \mathrm{O}$ (1 equiv.), nitrogen protection, at different tempertures. ${ }^{b}$ Yield of isolated product.

在优化的条件下，嫝可与邻苯二胺反应成功转化为 环状嫝的衍生物(表 2, Entry 12), 这种环状脲产物在生 物学领域具有一定的研究价值 ${ }^{[32]}$. 有趣的是, 1-苯基嫝 与对甲苯胺之间的反应得到的产物只有一种, 即 1-苯基 脲的羰基两侧均被取代的产物 1,3-二对甲苯基脲(表 2, Entry 14). 其次, 嫝与苯胺以及 1,3-二苯基嫝与苠胺之 间的反应得到的产物也是羰基两侧均被取代的对称产 物(表 2, Entries 13,15).

在以苯乙酰胺为底物的反应中, 重点考察了苯胺芳 环上取代基效应对反应的影响. 结果显示, 甲氧基苯胺 和苯胺的反应活性比对氟苯胺稍高一些, 没有表现出由 于取代基电子效应而引起的较大的差异(表 2, Entries $16 \sim 18)$. 此外, 在以苯甲酰胺为底物的反应中, 苯胺和 苠胺也表现出较高的反应活性(表 2, Entries 19,20).

$(R)$-乳酰胺可与对甲苯胺、苯胺及对氯苯胺分别发 生氨解反应, 得到 $(R)$-乳酰胺的氨基被取代的产物(表 2 , Entries 21 23), 在反应过程中 $(R)$-乳酰胺的光学活性基 才没有发生消旋化. 其中, 对氯苯胺分子中苯环上氯原 子的静态诱导效应的拉电子作用, 降低了苯胺的氨基亲 核取代能力, 造成对氯苯胺与 $(R)$-乳酰胺的反应时间较 长, 预期产物收率较低.
将底物扩展到杂环取代的酰胺衍生物, 研究结果显 示带有吡啶基团的酰胺也能与苯胺及其衍生物发生氨 解反应, 并得到令人满意的收率(表 2, Entries 24 26).

尝试了乳酸催化下邻苯二甲酰亚胺与苄胺之间的 反应(表 2 , Entry 27), 令人失望的是它们之间没有任何 的预期产物生成，这与文献报道的磺化铇酸盐、硼酸催 化的实验结果大相径庭 ${ }^{[15-16]}$, 但造成乳酸不能催化邻苯 二甲酰亚胺与苄胺之间发生反应的原因尚不清楚 (Scheme 1).

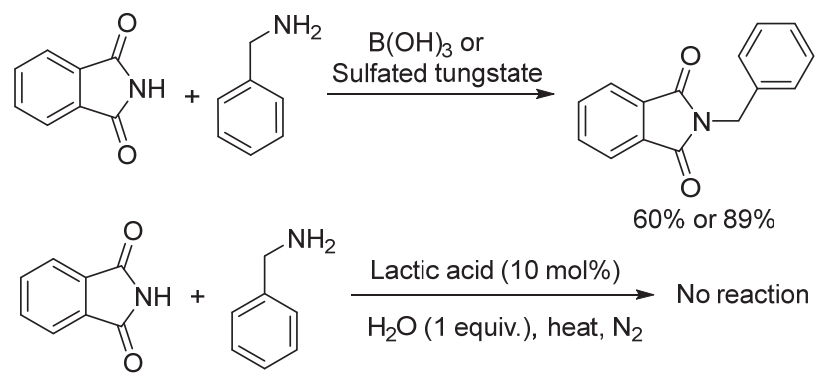

图式 1 磺化钨酸盐、嗍酸、乳酸催化邻苯二甲酰亚胺与苄胺 之间的反应

Scheme 1 Sulfated tungstate, boric acid, and lactic acid catalyzed reactions of phthalimide with benzylamine 
为了与其他文献报道的催化剂在 $N$-芐基甲酰胺合 成中的催化效果进行对比, 在表 3 中列出了各种催化剂 在加入 $\mathrm{H}_{2} \mathrm{O}$ (1 equiv.), $130{ }^{\circ} \mathrm{C}$ 相同反应条件下合成目标 产物 $3 \mathbf{t}$ 的产率. 由表 3 可以很明显地看出, 选用乳酸作 为催化剂时产品收率较高, 显示出了一定的优势.

表 3 不同催化剂催化合成化合物 $3 t$ 效果对比

Table 3 Comparison of the effect of catalysts for the synthesis of $3 \mathbf{t}$

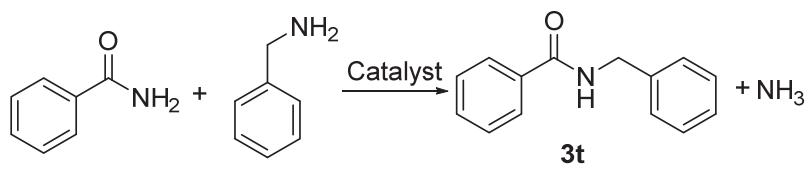

\begin{tabular}{clccc}
\hline Entry & \multicolumn{1}{c}{ Catalyst (loading) } & Time/h & Yield ${ }^{a} \%$ & Ref. \\
\hline 1 & Lactic acid $(10 \mathrm{~mol} \%)$ & 11 & 97 & - \\
2 & $\mathrm{H}_{2} \mathrm{NOH} \cdot \mathrm{HCl}(10 \mathrm{~mol} \%)$ & 11 & 20 & {$[18]$} \\
3 & Sulfated tungstate $(10 \mathrm{~mol} \%)$ & 11 & 86 & {$[15]$} \\
4 & $\mathrm{~B}(\mathrm{OH})_{3}(10 \mathrm{~mol} \%)$ & 11 & 34 & {$[17]$} \\
5 & $\mathrm{Fe}\left(\mathrm{NO}_{3}\right){ }_{3} \cdot 9 \mathrm{H}_{2} \mathrm{O}(10 \mathrm{~mol} \%)$ & 11 & 46 & {$[14]$} \\
6 & Benzoic acid $(10 \mathrm{~mol} \%)$ & 11 & 13 & {$[19]$} \\
7 & L-Proline $(10 \mathrm{~mol} \%)$ & 11 & 82 & {$[20]$} \\
8 & Benzotriazole $(10 \mathrm{~mol} \%)$ & 11 & 83 & {$[21]$} \\
9 & Chitosan $(10 \mathrm{~mol} \%)$ & 11 & $\mathrm{NR}$ & {$[22]$} \\
\hline
\end{tabular}

${ }^{a}$ Isolated yields.

为了评估该方法在大规模反应中的可行性，进行了 放大实验，将甲酰胺 $(0.45 \mathrm{~g}, 10 \mathrm{mmol}) 、$ 芐胺 $(1.07 \mathrm{~g}, 10$ $\mathrm{mmol})$ 在乳酸 $(0.11 \mathrm{~g}, 1 \mathrm{mmol})$ 与水 $(0.02 \mathrm{~g}, 1 \mathrm{mmol})$ 催化下 进行反应, 其收率几乎与小规模反应的收率相同(Eq. 2).

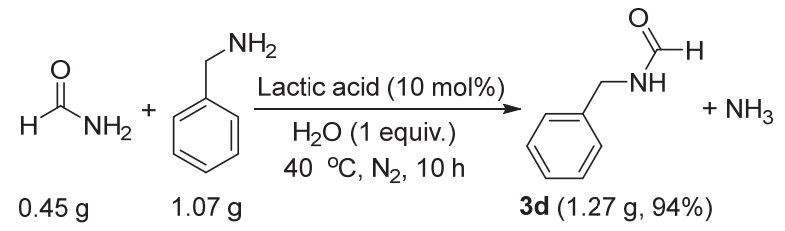

\section{3 反应机理}

在本文实验结果及文献报道的相关反应机理的基 础上 ${ }^{[11,14,17-19]}$, 推测了乳酸催化下酰胺的氨解反应可能 的反应机理(Scheme 2). 首先, 催化剂乳酸中羧基上的 氢与酰胺 1 上带有未成键孤对电子的氮原子相互作用, 形成分子间氢键, 同时乳酸中羟基上的氢原子与酰胺中 羰基上带有未成键孤对电子的氧原子相互作用, 形成分 子间氢键, 进而形成九元环的中间体 A. 其次, 胺 2 中 带有孤对电子的氮原子进攻中间体 $\mathbf{A}$ 中原酰胺中羰基 上的碳原子, 而胺中的一个氢原子转移到原酰胺中羰基 的氧原子上，同时原分子间氢键(b)断裂，乳酸分子结构 中羟基上的氢原子与胺上的氮原子相互作用的形成新 的分子间氢键(c), 得到中间体 $\mathbf{B}$. 然后，在中间体 $\mathbf{B}$ 中, 原酰胺中的碳氮键断裂, 氢原子发生 1,3-迁移, 形成中
间体 C. 最后，中间体 $\mathbf{C}$ 中的氢键(a)和(c)断裂，则得到 新的酰胺 3 和氨分子，同时乳酸分子被释放出来，重新 参与催化下一轮反应. 这与 Nguyen ${ }^{[17]} 、 A l l e n^{[18]} 、 \mathrm{Wu}^{[19]}$ 等所推测的反应机理一致: 乳酸与酰胺形成两点接触的 分子间氢键, 酰胺与胺的氨解反应能够在这种氢键的诱 导作用下顺利地进行.

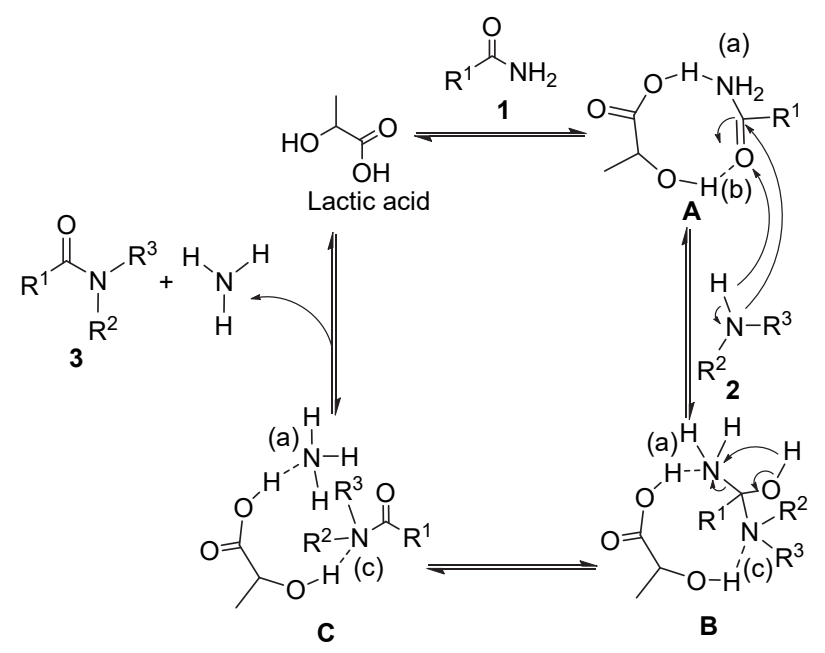

图式 2 乳酸催化酰胺与氨之间氨解的可能反应机理

Scheme 2 Possible mechanism for lactic acid-catalyzed transamidation of carboxamides with amines

\section{2 结论}

总之, 在无溶剂条件下使用乳酸作为催化剂发展了 一种酰胺与氨的氨解反应新方法, 且利用该方法成功合 成了一系列芳香、脂肪类酰胺和脲的衍生物. 该方法最 显著的特征是催化剂廉价易得、过程环保、转化率高及 操作简单, 为合成酰胺类化合物提供了一条新的途径.

\section{3 实验部分}

\section{1 仪器与试剂}

熔点测定使用北京泰克仪器有限公司 X-4 数字显示 显微熔点测试仪; ${ }^{1} \mathrm{H}$ NMR 和 ${ }^{13} \mathrm{C}$ NMR 采用瑞士 Bruker AVANCE-300 MHz 型、Bruker AVANCE-400 MHz 型、 Bruker AVANCE-500 MHz 型核磁共振仪测定, 以 $\mathrm{CDCl}_{3} 、 \mathrm{DMSO}-d_{6}$ 为溶剂; 高分辨质谱采用安捷伦 6520 Q-TOF LC/MS 仪器测定; 比旋光度采用数字自动旋光 仪 WZZ-2S/2SS, 在室温下使用容量为 $1 \mathrm{~mL}$ 长度为 10 $\mathrm{cm}$ 的盛液管, 溶液浓度为 $\mathrm{g} / 100 \mathrm{~mL}$, 钠光灯作为光源; 高效液相色谱分析使用 Agilent 1100 系列系统, 并使用 相应手性柱进行测试，峰面积是通过使用 JASCO chrom NAV (Windows 7)作为自动积分器计算得到的. 除特别 说明外, 本文所用化学试剂均为市售化学纯或分析纯, 所购买的试剂无需进一步纯化, 直接使用. 柱层析硅胶 (试剂级, 200 300 目)及 GF254 硅胶板均购自青岛海洋 
化工有限公司, 直接使用. 所用的展开剂、淋洗剂为石 油醚(b.p. 60 90 ${ }^{\circ}$ C) 和乙酸乙酯, 重蒸后使用. 所有反 应均在 $\mathrm{N}_{2}$ 保护下采取无氧操作, 添加剂水为自来水, 无 需使用蒸馏水或超纯水. 反应加热控温使用控温油浴 锅, 用薄层色谱(TLC)方法跟踪监测反应的进程.

\section{2 实验方法}

在清洁干燥的圆底烧瓶中依次加入胺 $(1.0 \mathrm{mmol})$, 酰 胺 $(1.0 \mathrm{mmol})$, 催化剂乳酸 $(0.1 \mathrm{mmol})$ 和添加剂水 $(0.1$ $\mathrm{mmol})$, 反应混合物在 $\mathrm{N}_{2}$ 保护下加热至所需温度, TLC 跟 踪监测直至反应结束, 停止反应. 将反应体系冷却至室 温, 加入 $2.0 \mathrm{~mL}$ 水搅拌, 再加入 $2.0 \mathrm{~mL}$ 二氯甲烷萃取, 水洗(2.0 mL $\times 3)$, 分液, 收集有机相, 无水硫酸钠干燥, 过滤, 蒸除溶剂, 粗产品用柱色谱法进行分离提纯, 得到 纯净的产物 $3 \mathrm{a} \sim \mathbf{3 z}$.

已知化合物 3a 3n、3p 3r、3t 3v、3 的结构由 熔点、 ${ }^{1} \mathrm{H} N M R 、{ }^{13} \mathrm{C}$ NMR 数据与文献数据进行比较后 确定. 未知化合物 $30 、 3 \mathrm{~s} 、 3 \mathrm{w} 、 3 \mathrm{y} 、 3 \mathrm{z}$ 的结构通过 ${ }^{1} \mathrm{H} N M R$ 、 ${ }^{13} \mathrm{C}$ NMR 和 HRMS 确定.

4-羟基苯甲酰胺(3a): 深褐色固体. m.p. 131 $133{ }^{\circ} \mathrm{C}$ (Lit. $\left.{ }^{[17]} 132 \sim 135{ }^{\circ} \mathrm{C}\right)$; ${ }^{1} \mathrm{H}$ NMR $(300 \mathrm{MHz}$, DMSO- $\left.d_{6}\right) \delta: 9.92(\mathrm{~s}, 1 \mathrm{H}), 8.16(\mathrm{~s}, 1 \mathrm{H}), 7.39$ (d, $J=5.7$ $\mathrm{Hz}, 2 \mathrm{H}), 6.72$ (d, $J=5.1 \mathrm{~Hz}, 1 \mathrm{H}), 3.92$ (s, 1H); ${ }^{13} \mathrm{C} \mathrm{NMR}$ $\left(75 \mathrm{MHz}, \mathrm{DMSO}-d_{6}\right) \delta: 162.5,158.8,154.2,153.5,129.9$, 120.7, 120.1, 115.8, 115.1.

$N$-(4-氯苯基)甲酰胺(3b): 白色固体. m.p. 102 $104{ }^{\circ} \mathrm{C}$ (Lit. ${ }^{[17]} 103 \sim 105{ }^{\circ} \mathrm{C}$ ); ${ }^{1} \mathrm{H}$ NMR $(300 \mathrm{MHz}$, DMSO- $\left.d_{6}\right) \delta: 10.35$ (br s, $\left.1 \mathrm{H}\right), 8.81(\mathrm{~d}, J=8.1 \mathrm{~Hz}, 1 \mathrm{H})$, 8.30 (d, $J=1.2 \mathrm{~Hz}, 1 \mathrm{H}), 7.64$ (d, $J=5.7 \mathrm{~Hz}, 2 \mathrm{H}), 7.39$ (d, $J=6.6 \mathrm{~Hz}, 2 \mathrm{H}), 7.24(\mathrm{~d}, J=6.6 \mathrm{~Hz}, 2 \mathrm{H}), 3.41(\mathrm{~s}, 1 \mathrm{H}) ;{ }^{13} \mathrm{C}$ NMR (75 MHz, DMSO- $\left.d_{6}\right) \delta$ : 163.0, 160.2, 137.6, 129.2, $127.9,121.2,119.4$.

4'-甲氧基甲酰苯胺(3c): 白色固体. m.p. $79 \sim 81{ }^{\circ} \mathrm{C}$ (Lit. $\left.{ }^{[15]} 75 \sim 78{ }^{\circ} \mathrm{C}\right) ;{ }^{1} \mathrm{H}$ NMR $\left(500 \mathrm{MHz}, \mathrm{DMSO}-d_{6}\right) \delta$ : 10.00 (br s, $1 \mathrm{H}), 8.61$ (d, $J=10.8 \mathrm{~Hz}, 1 \mathrm{H}), 8.21$ (d, $J=1.2$ $\mathrm{Hz}, 1 \mathrm{H}), 7.52$ (d, $J=6.6 \mathrm{~Hz}, 2 \mathrm{H}), 7.13$ (d, $J=6.9 \mathrm{~Hz}, 2 \mathrm{H})$, $6.90(\mathrm{~d}, J=6.6 \mathrm{~Hz}, 2 \mathrm{H}), 3.72(\mathrm{~s}, 3 \mathrm{H}), 3.39(\mathrm{~s}, 1 \mathrm{H}) ;{ }^{13} \mathrm{C}$ NMR (125 MHz, DMSO- $\left.d_{6}\right) \delta: 163.0,159.5,155.9,131.9$, 121.1, 114.4, 55.6.

$N$-苄基甲酰胺 $(3 d)$ : 白色粉末. m.p. $60 \sim 61{ }^{\circ} \mathrm{C}$ (Lit. $\left.{ }^{[18]} 65 \sim 68{ }^{\circ} \mathrm{C}\right) ;{ }^{1} \mathrm{H}$ NMR $\left(300 \mathrm{MHz}, \mathrm{CDCl}_{3}\right) \delta: 8.18$ (br s, $1 \mathrm{H}), 8.10(\mathrm{~d}, J=9.0 \mathrm{~Hz}, 1 \mathrm{H}), 7.38 \sim 7.22(\mathrm{~m}, 5 \mathrm{H})$, 6.42 (br s, 1H), 4.43 (d, $J=4.5 \mathrm{~Hz}, 2 \mathrm{H}) ;{ }^{13} \mathrm{C}$ NMR $(75$ $\left.\mathrm{MHz}, \mathrm{CDCl}_{3}\right) \delta: 164.8,161.3,137.6,128.9,128.8,127.8$, 127.6, 127.0, 45.7, 42.1.

$N$-甲酰吗啉 $(\mathbf{3 e})^{[17]}$ : 无色油状. ${ }^{1} \mathrm{H}$ NMR (300 MHz,
$\left.\mathrm{CDCl}_{3}\right) \delta: 7.92(\mathrm{~s}, 1 \mathrm{H}), 3.57(\mathrm{t}, J=2.7 \mathrm{~Hz}, 2 \mathrm{H}), 3.53(\mathrm{t}$, $J=2.7 \mathrm{~Hz}, 2 \mathrm{H}), 3.43(\mathrm{t}, J=2.7 \mathrm{~Hz}, 2 \mathrm{H}), 3.28(\mathrm{t}, J=2.7$ $\mathrm{Hz}, 2 \mathrm{H}) ;{ }^{13} \mathrm{C} \mathrm{NMR}\left(75 \mathrm{MHz}, \mathrm{CDCl}_{3}\right) \delta: 161.7,67.8,67.0$, 46.4, 41.2 .

$N$-甲基- $N$-苯基甲酰胺 $(3 \mathbf{f})^{[17]}$ : 棕色油状. ${ }^{1} \mathrm{H}$ NMR $\left(300 \mathrm{MHz}, \mathrm{CDCl}_{3}\right) \delta: 8.47(\mathrm{~s}, 1 \mathrm{H}), 7.43 \sim 7.36(\mathrm{~m}, 2 \mathrm{H})$, $7.30 \sim 7.23(\mathrm{~m}, 1 \mathrm{H}), 7.19 \sim 7.10(\mathrm{~m}, 2 \mathrm{H}), 3.32(\mathrm{~s}, 3 \mathrm{H}) ;{ }^{13} \mathrm{C}$ NMR (75 MHz, $\left.\mathrm{CDCl}_{3}\right) \delta: 162.4,142.1,129.6,126.4$, $122.3,32.0$.

$N$-甲酰基哌啶 $(\mathbf{3 g})^{[17]}$ : 无色油状. ${ }^{1} \mathrm{H}$ NMR (300 $\left.\mathrm{MHz} \mathrm{CDCl}_{3}\right) \delta: 8.01(\mathrm{~s}, 1 \mathrm{H}), 3.49(\mathrm{t}, J=3.6 \mathrm{~Hz}, 2 \mathrm{H}), 3.34$ $(\mathrm{t}, J=3.6 \mathrm{~Hz}, 2 \mathrm{H}), 1.70 \sim 1.62(\mathrm{~m}, 2 \mathrm{H}), 1.60 \sim 1.56(\mathrm{~m}$, $4 \mathrm{H}) ;{ }^{13} \mathrm{C} \mathrm{NMR}\left(75 \mathrm{MHz}, \mathrm{CDCl}_{3}\right) \delta: 160.8,46.8,40.6,26.6$, 25.1, 24.7.

$N$-环己基甲酰胺(3h) ${ }^{[15]}$ : 棕色油状. ${ }^{1} \mathrm{H}$ NMR (300 $\left.\mathrm{MHz}, \mathrm{CDCl}_{3}\right) \delta: 8.08($ br s, $1 \mathrm{H}), 3.83 \sim 3.72(\mathrm{~m}, 1 \mathrm{H})$, $1.90 \sim 1.17(\mathrm{~m}, 11 \mathrm{H}) ;{ }^{13} \mathrm{C} \mathrm{NMR}\left(75 \mathrm{MHz}, \mathrm{CDCl}_{3}\right) \delta:$ 163.9, 160.7, 51.2, 47.1, 34.6, 32.9, 25.4, 25.0, 24.78.

$\mathrm{N}$-(2-苯基乙基)-甲酰胺(3i) ${ }^{[17]}$ : 无色油状. ${ }^{1} \mathrm{H}$ NMR $\left(300 \mathrm{MHz}, \mathrm{CDCl}_{3}\right) \delta: 8.04$ (br s, $\left.1 \mathrm{H}\right), 7.81$ (d, $J=9.0 \mathrm{~Hz}$, $1 \mathrm{H}$ ), $7.31 \sim 7.15$ (m, 5H), 6.25 (br s, $1 \mathrm{H}), 3.55$ (q, $J=5.1$ $\mathrm{Hz}, 2 \mathrm{H}), 3.45$ (q, $J=5.1 \mathrm{~Hz}, 2 \mathrm{H}), 2.83$ (t, $J=5.4 \mathrm{~Hz}, 2 \mathrm{H})$; ${ }^{13} \mathrm{C}$ NMR (75 MHz, $\left.\mathrm{CDCl}_{3}\right) \delta: 164.8,161.5,138.6,137.7$, 128.9, 128.8, 128.7, 126.6, 43.3, 39.2, 37.7, 35.5.

$(S)-N$-(1-苯基乙基)-甲酰胺 $(\mathbf{3 j})^{[17]}$ : 无色油状. $[\alpha]_{\mathrm{D}}^{25}$ -36.8 (c 1, $\left.\mathrm{CHCl}_{3}\right) ;{ }^{1} \mathrm{H}$ NMR (300 $\left.\mathrm{MHz}, \mathrm{CDCl}_{3}\right) \delta: 8.01$ $(\mathrm{s}, 1 \mathrm{H}), 7.33 \sim 7.21(\mathrm{~m}, 5 \mathrm{H}), 5.12(\mathrm{q}, J=2.7 \mathrm{~Hz}, 1 \mathrm{H}), 1.44$ $(\mathrm{d}, J=5.1 \mathrm{~Hz}, 3 \mathrm{H}) ;{ }^{13} \mathrm{C} \mathrm{NMR}\left(75 \mathrm{MHz}, \mathrm{CDCl}_{3}\right) \delta: 160.7$, 142.8, 128.6, 127.3, 126.1, 47.6, 21.8.

$N$-苠基乙酰胺 (3k): 白色晶体. m.p. 59 60 ${ }^{\circ} \mathrm{C}$ (Lit. $\left.{ }^{[18]} 62 \sim 65{ }^{\circ} \mathrm{C}\right) ;{ }^{1} \mathrm{H}$ NMR $\left(300 \mathrm{MHz}, \mathrm{CDCl}_{3}\right) \delta$ : $7.36 \sim 7.26(\mathrm{~m}, 5 \mathrm{H}), 5.89(\mathrm{~s}, 1 \mathrm{H}), 4.43(\mathrm{~d}, J=4.2 \mathrm{~Hz}, 2 \mathrm{H})$, $2.02(\mathrm{~s}, 3 \mathrm{H}) ;{ }^{13} \mathrm{C} \mathrm{NMR}\left(75 \mathrm{MHz}, \mathrm{CDCl}_{3}\right) \delta: 169.9,138.2$, 128.7, 127.9, 127.6, 43.8, 23.3.

2-苯并咪唑啉酮(3I): 白色固体. m.p. $304 \sim 307{ }^{\circ} \mathrm{C}$ (Lit. $\left.{ }^{[11]} 305 \sim 308{ }^{\circ} \mathrm{C}\right) ;{ }^{1} \mathrm{H}$ NMR (300 MHz, DMSO- $\left.d_{6}\right) \delta$ : $10.64(\mathrm{~s}, 2 \mathrm{H}), 6.95(\mathrm{~s}, 4 \mathrm{H}) ;{ }^{13} \mathrm{C}$ NMR (75 MHz, DMSO- $\left.d_{6}\right)$ $\delta: 156.5,130.8,121.6,109.7$.

1,3-二苯基脲 $(\mathbf{3 m})$ : 白色固体. m.p. $235 \sim 238{ }^{\circ} \mathrm{C}$; (Lit. $\left.{ }^{[11]} 234 \sim 237{ }^{\circ} \mathrm{C}\right) ;{ }^{1} \mathrm{H}$ NMR (300 MHz, DMSO- $\left.d_{6}\right) \delta$ : 8.67 (s, 2H), 7.48 (d, $J=5.7 \mathrm{~Hz}, 4 \mathrm{H}), 7.31$ (t, $J=5.7 \mathrm{~Hz}$, $4 \mathrm{H}), 6.99(\mathrm{t}, J=5.7,2 \mathrm{H}) ;{ }^{13} \mathrm{C} \mathrm{NMR}\left(75 \mathrm{MHz}, \mathrm{DMSO}-d_{6}\right)$ $\delta: 153.0,140.2,129.3,122.3,118.6$.

1,3-二(4-甲基苯基)脲(3n): 白色固体. m.p. 267 $269{ }^{\circ} \mathrm{C}$ (Lit. $\left.{ }^{[11]} 263 \sim 265{ }^{\circ} \mathrm{C}\right) ;{ }^{1} \mathrm{H}$ NMR $(300 \mathrm{MHz}$, 
DMSO- $\left.d_{6}\right) \delta: 8.46(\mathrm{~s}, 2 \mathrm{H}), 7.34(\mathrm{~d}, J=10.8 \mathrm{~Hz}, 4 \mathrm{H}), 7.07$ (d, $J=11.2 \mathrm{~Hz}, 4 \mathrm{H}), 2.24$ (s, 6H); ${ }^{13} \mathrm{C}$ NMR $(75 \mathrm{MHz}$, DMSO- $\left.d_{6}\right) \delta: 152.6,137.2,130.5,129.1,118.2,20.3$.

1,3-二苄基嫝(3o): 白色晶体. m.p. $175 \sim 177{ }^{\circ} \mathrm{C} ;{ }^{1} \mathrm{H}$ NMR (400 MHz, DMSO- $\left.d_{6}\right) \delta: 7.31 \sim 7.19(\mathrm{~m}, 10 \mathrm{H}), 6.38$ (t, $J=6.0,2 \mathrm{H}), 3.90$ (d, $J=16.0 \mathrm{~Hz}, 4 \mathrm{H}) ;{ }^{13} \mathrm{C}$ NMR $(100$ $\left.\mathrm{MHz}, \mathrm{CDCl}_{3}\right) \delta: 133.8,123.4,122.2,122.1,39.3$; HRMS calcd for $\mathrm{C}_{15} \mathrm{H}_{16} \mathrm{~N}_{2} \mathrm{O} 240.3003$, found 240.3009 .

$N$-苯基-2-苯基乙酰胺 (3p): 白色晶体. m.p. 117 $119{ }^{\circ} \mathrm{C}$ (Lit. $\left.{ }^{[1]} 110 \sim 112{ }^{\circ} \mathrm{C}\right)$; ${ }^{1} \mathrm{H}$ NMR $(300 \mathrm{MHz}$, $\left.\mathrm{CDCl}_{3}\right) \delta: 7.42 \sim 7.26(\mathrm{~m}, 9 \mathrm{H}), 7.15(\mathrm{br} \mathrm{s}, 1 \mathrm{H}), 7.10(\mathrm{t}, J=$ $5.6 \mathrm{~Hz}, 1 \mathrm{H}), 3.74(\mathrm{~s}, 2 \mathrm{H}) ;{ }^{13} \mathrm{C} \mathrm{NMR}\left(75 \mathrm{MHz}, \mathrm{CDCl}_{3}\right) \delta$ : $169.2,137.6,134.5,129.6,129.3,129.0,127.7,124.5$, $119.9,44.9$.

$\mathrm{N}$-(4-氟苯基)-2-苯基乙酰胺 (3q): 白色固体. m.p. $124 \sim 126{ }^{\circ} \mathrm{C}$ (Lit. ${ }^{[1]} 123 \sim 125{ }^{\circ} \mathrm{C}$ ); ${ }^{1} \mathrm{H}$ NMR $(300 \mathrm{MHz}$, $\left.\mathrm{CDCl}_{3}\right) \delta: 7.42 \sim 7.32(\mathrm{~m}, 7 \mathrm{H}), 7.16(\mathrm{br} \mathrm{s}, 1 \mathrm{H}), 6.98(\mathrm{t}, J=$ $6.4 \mathrm{~Hz}, 2 \mathrm{H}), 3.72(\mathrm{~s}, 2 \mathrm{H}) ;{ }^{13} \mathrm{C} \mathrm{NMR}\left(75 \mathrm{MHz}, \mathrm{CDCl}_{3}\right) \delta$ : $169.2,160.7,158.2,134.3,129.6,129.3,127.8,121.8$, $121.7,115.7,115.5,44.7$.

$\mathrm{N}$-(4-甲氧基苯基)-2-苯基乙酰胺(3r): 白色晶体. m.p. $125 \sim 126{ }^{\circ} \mathrm{C}$ (Lit. $\left.{ }^{[17]} 125 \sim 128{ }^{\circ} \mathrm{C}\right) ;{ }^{1} \mathrm{H}$ NMR $(300$ $\left.\mathrm{MHz}, \mathrm{CDCl}_{3}\right) \delta: 7.42 \sim 7.30(\mathrm{~m}, 7 \mathrm{H}), 7.06(\mathrm{~s}, 1 \mathrm{H}), 6.81(\mathrm{~d}$, $J=9.6 \mathrm{~Hz}, 2 \mathrm{H}), 3.76$ (s, 3H), 3.72 (s, 2H); ${ }^{13} \mathrm{C}$ NMR $(75$ $\left.\mathrm{MHz}, \mathrm{CDCl}_{3}\right) \delta: 169.0,156.5,134.6,130.7,129.6,129.2$, 127.6, 121.8, 114.0, 55.4, 44.6.

$N$-苯基苯甲酰胺(3s): 白色晶体. m.p. $165 \sim 167{ }^{\circ} \mathrm{C}$ $\left(\right.$ Lit. $\left.{ }^{[18]} 165 \sim 168{ }^{\circ} \mathrm{C}\right) ;{ }^{1} \mathrm{H}$ NMR $\left(400 \mathrm{MHz}, \mathrm{CDCl}_{3}\right) \delta$ : 7.90 (br s, 1H, NH), 7.87 (d, $J=6.6 \mathrm{~Hz}, 2 \mathrm{H}), 7.65$ (d, $J=$ $5.7 \mathrm{~Hz}, 2 \mathrm{H}), 7.57(\mathrm{t}, J=5.4 \mathrm{~Hz}, 1 \mathrm{H}), 7.50(\mathrm{t}, J=5.6 \mathrm{~Hz}$, $2 \mathrm{H}), 7.39(\mathrm{t}, J=6.0 \mathrm{~Hz}, 2 \mathrm{H}), 7.17(\mathrm{t}, J=5.6 \mathrm{~Hz}, 1 \mathrm{H}) ;{ }^{13} \mathrm{C}$ NMR $\left(100 \mathrm{MHz}, \mathrm{CDCl}_{3}\right) \delta: 165.8,137.9,135.0,131.9$, 129.1, 128.8, 127.0, 124.6, 120.2; HRMS calcd for $\mathrm{C}_{13} \mathrm{H}_{12} \mathrm{NO}(\mathrm{M}+\mathrm{H})^{+}$198.0969, found 198.0961.

$N$-芐基苯甲酰胺(3t): 白色固体. m.p. $101 \sim 102{ }^{\circ} \mathrm{C}$ (Lit. $\left.{ }^{[18]} 105 \sim 108{ }^{\circ} \mathrm{C}\right)$; ${ }^{1} \mathrm{H}$ NMR $\left(300 \mathrm{MHz}, \mathrm{CDCl}_{3}\right) \delta$ : $7.80(\mathrm{~d}, J=5.4 \mathrm{~Hz}, 2 \mathrm{H}), 7.50 \sim 7.25(\mathrm{~m}, 9 \mathrm{H}), 4.63(\mathrm{~d}, J=$ $4.5 \mathrm{~Hz}, 2 \mathrm{H}) ;{ }^{13} \mathrm{C}$ NMR $\left(75 \mathrm{MHz}, \mathrm{CDCl}_{3}\right) \delta: 168.2,138.9$, 135.1, 132.3, 129.5, 129.3, 128.6, 128.3, 127.7, 44.8.

(R)-2-羊基- $N$-对甲苯基丙酰胺 (3u): 白色固体. m.p. $92 \sim 93{ }^{\circ} \mathrm{C} \quad\left(\right.$ Lit. $\left.^{[11]} 84 \sim 86{ }^{\circ} \mathrm{C}\right) ;[\alpha]_{\mathrm{D}}^{25}+41.0(c \quad 0.5$, $\mathrm{MeOH}) ;{ }^{1} \mathrm{H}$ NMR $\left(300 \mathrm{MHz}, \mathrm{CDCl}_{3}\right) \delta: 8.50(\mathrm{~s}, 1 \mathrm{H}), 7.44$ (d, $J=6.3 \mathrm{~Hz}, 2 \mathrm{H}), 7.14$ (d, $J=6.3 \mathrm{~Hz}, 2 \mathrm{H}), 4.36$ (q, $J=$ $5.1 \mathrm{~Hz}, 1 \mathrm{H}), 2.33$ (s, 3H), $1.52(\mathrm{~d}, J=5.1 \mathrm{~Hz}, 3 \mathrm{H}) ;{ }^{13} \mathrm{C}$ NMR (75 MHz, $\left.\mathrm{CDCl}_{3}\right) \delta: 172.7,134.6,134.3,129.5$,
119.9, 68.7, 21.2, 20.9.

(R)-2-羟基- $N$-苯基丙酰胺 (3v): 白色固体. m.p. 60 $62{ }^{\circ} \mathrm{C}$ (Lit. $\left.{ }^{[11]} 55 \sim 57{ }^{\circ} \mathrm{C}\right) ;[\alpha]_{\mathrm{D}}^{25}+38.6(c \quad 0.5, \mathrm{MeOH})$; ${ }^{1} \mathrm{H}$ NMR (400 MHz, $\left.\mathrm{CDCl}_{3}\right) \delta: 8.40(\mathrm{~s}, 1 \mathrm{H}), 7.48$ (d, $J=$ $8.0 \mathrm{~Hz}, 2 \mathrm{H}), 7.29$ (s, 1H), 7.17 (d, $J=8.0,2 \mathrm{H}), 4.42$ (q, $J=6.8 \mathrm{~Hz}, 1 \mathrm{H}), 2.35$ (br s, $1 \mathrm{H}), 1.45$ (d, $J=6.8 \mathrm{~Hz}, 3 \mathrm{H}$ ); ${ }^{13} \mathrm{C} \mathrm{NMR}\left(100 \mathrm{MHz}, \mathrm{CDCl}_{3}\right) \delta: 172.1,134.6,134.2,129.5$, $119.8,68.9,21.3,20.9$.

(R)-2-羟基- $\mathrm{N}$-(4-氯苯基)丙酰胺(3w): 白色固体. m.p. $102 \sim 104{ }^{\circ} \mathrm{C} ;[\alpha]_{\mathrm{D}}^{25}+43.0(c 0.5, \mathrm{MeOH}) ;{ }^{1} \mathrm{H} \mathrm{NMR}$ $\left(400 \mathrm{MHz}, \mathrm{CDCl}_{3}\right) \delta: 8.54(\mathrm{~s}, 1 \mathrm{H}), 7.51(\mathrm{~d}, J=8.4 \mathrm{~Hz}$, $2 \mathrm{H}), 7.29(\mathrm{~d}, J=10.0 \mathrm{~Hz}, 2 \mathrm{H}), 4.39(\mathrm{q}, J=6.8 \mathrm{~Hz}, 1 \mathrm{H})$, 2.90 (br s, $1 \mathrm{H}), 1.53$ (d, $J=6.8 \mathrm{~Hz}, 3 \mathrm{H}) ;{ }^{13} \mathrm{C}$ NMR $(100$ $\left.\mathrm{MHz}, \mathrm{CDCl}_{3}\right) \delta: 172.5,135.7,129.6,129.1,121.0,68.9$, 21.2; HRMS calcd for $\mathrm{C}_{9} \mathrm{H}_{10} \mathrm{ClNO}_{2}$ 199.6342, found 199.6337.

$\mathrm{N}$-苯基烟酰胺(3x): 白色固体. m.p. 123 126 ${ }^{\circ} \mathrm{C}$ (Lit. ${ }^{[19]} 125 \sim 127{ }^{\circ} \mathrm{C}$ ); ${ }^{1} \mathrm{H}$ NMR (400 MHz, $\left.\mathrm{CDCl}_{3}\right) \delta$ : $9.09(\mathrm{~s}, 1 \mathrm{H}), 8.73(\mathrm{~d}, J=4.0 \mathrm{~Hz}, 1 \mathrm{H}), 8.35(\mathrm{~s}, 1 \mathrm{H}), 8.20$ (d, $J=7.6 \mathrm{~Hz}, 1 \mathrm{H}), 7.65(\mathrm{~d}, J=7.6 \mathrm{~Hz}, 2 \mathrm{H}), 7.42 \sim 7.35$ (m, 3H), 7.19 (t, $J=7.4 \mathrm{~Hz}, 1 \mathrm{H}) ;{ }^{13} \mathrm{C}$ NMR $(100 \mathrm{MHz}$, $\left.\mathrm{CDCl}_{3}\right) \delta: 164.0,152.3,147.8,137.5,135.5,130.9,129.2$, 125.1, 123.7, 120.5.

$N$-(4-澳苯基)烟酰胺(3y)：白色固体. m.p. 183 $186{ }^{\circ} \mathrm{C} ;{ }^{1} \mathrm{H}$ NMR $\left(400 \mathrm{MHz}, \mathrm{CDCl}_{3}\right) \delta: 9.14(\mathrm{~s}, 1 \mathrm{H}), 8.82$ (d, $J=2.0 \mathrm{~Hz}, 1 \mathrm{H}), 8.26$ (d, $J=8.0 \mathrm{~Hz}, 1 \mathrm{H}), 8.08(\mathrm{~s}, 1 \mathrm{H})$, $7.60 \sim 7.48(\mathrm{~m}, 5 \mathrm{H}) ;{ }^{13} \mathrm{C}$ NMR $\left(100 \mathrm{MHz}, \mathrm{CDCl}_{3}\right) \delta$ : $152.6,147.7,136.5,135.6,132.2,123.9,122.0,117.8$. HRMS calcd for $\mathrm{C}_{12} \mathrm{H}_{9} \mathrm{BrN}_{2} \mathrm{O} 277.1167$, found 277.1173.

$N$-(4-氯苯基)异烟酰胺 (3z): 白色固体. m.p. 156 $159{ }^{\circ} \mathrm{C} ;{ }^{1} \mathrm{H}$ NMR $\left(400 \mathrm{MHz}, \mathrm{CDCl}_{3}\right) \delta: 8.72$ (d, $J=4.8$ $\mathrm{Hz}, 2 \mathrm{H}), 8.64$ (s, 1H), 7.70 (d, $J=5.2 \mathrm{~Hz}, 2 \mathrm{H}), 7.62(\mathrm{~d}, J=$ $8.4 \mathrm{~Hz}, 2 \mathrm{H}), 7.35(\mathrm{~d}, J=8.8 \mathrm{~Hz}, 2 \mathrm{H}) ;{ }^{13} \mathrm{C}$ NMR $(100 \mathrm{MHz}$, $\left.\mathrm{CDCl}_{3}\right) \delta: 164.0,150.5,141.9,135.9,130.4,129.2,121.8$, 121.0; HRMS calcd for $\mathrm{C}_{12} \mathrm{H}_{9} \mathrm{ClN}_{2} \mathrm{O}$ 232.6657, found 232.6637 .

辅助材料(Supporting Information) 化合物 $\mathbf{3 a} \sim \mathbf{3 z}$ 的 ${ }^{1} \mathrm{H}$ NMR, ${ }^{13} \mathrm{C}$ NMR 图谱及化合物 $\mathbf{3 j}, \mathbf{3 u}, \mathbf{3 v}$ 和 $\mathbf{3 w}$ 的光学 纯度数据. 这些材料可以免费从本刊网站(http://siocjournal.cn/)上下载.

\section{References}

[1] Carey, J. S.; Laffan, D.; Thomson, C.; Williams, M. T. Org. Biomol. Chem. 2006, 4, 2337.

[2] Constable, D. J. C. Green Chem. 2007, 9, 411.

[3] Wildman, S. A.; Crippen, G. M. J. Chem. Inf. Comput. Sci. 1999, 
39,868 .

[4] Bhattacharya, S. K.; Andrews, K.; Beveridge, R.; Cameron, K. O.; Chen, C.; Dunn, M.; Fernando, D. P.; Gao, H.; Hepworth, D.; Jackson, V. M.; Khot, V.; Kong, J.; Kosa, R. E.; Lapham, K.; Loria, P. M.; Londregan, A. T.; McClure, K. F.; Orr, S. T. M.; Patel, J.; Rose, C.; Saenz, J.; Stock, I. A.; Storer, G.; Van Volkenburg, M.; Vrieze, D.; Wang, G.; Xiao, J.; Zhang, Y. ACS Med. Chem. Lett. 2014, 5, 474.

[5] McCoull, W.; Barton, P.; Brown, A.; Bowker, S.; Cameron, J.; Clarke, D.; Davies, R.; Dossetter, A.; Ertan, A.; Fenwick, M.; Green, C.; Holmes, J.; Martin, N.; Masters, D.; Moore, J.; Newcombe, N.; Newton, C.; Pointon, H.; Robb, G. R.; Sheldon, C.; Stokes, S.; Morgan, D. J. Med. Chem. 2014, 57, 6128.

[6] (a) Montalbetti, C. A. G. N.; Falque, V. Tetrahedron 2005, 61, 10827.

(b) Larock, R. C. Comprehensive Organic Transformations, Wiley-VCH, Weinheim, 1999.

(c) Bailey, P. D.; Collier, I. D.; Morgan, K. M. Comprehensive Organic Functional Group Transformations, Vol. 5, Elsevier, Cambridge, 1995, p. 257.

(d) Valeur, E.; Bradley, M. Chem. Soc. Rev. 2009, 38, 606.

(e) Dunetz, J. R.; Magano, J.; Weisenburger, G. A. Org. Process Res. Dev. 2016, 20, 140 .

[7] (a) Cossy, J.; Pale-Grosdemange, C. Tetrahedron Lett. 1989, 30, 2771 .

(b) Gooben, L. J.; Ohlmann, D. M.; Lange, P. P. Synthesis 2009, 160.

(c) Comerford, J. W.; Clark, J. H.; Macquarrie, D. J.; Breeden, S.

W. Chem. Commun. 2009, 2562.

(d) Ishihara, K.; Ohara, S.; Yamamoto, H. J. Org. Chem. 1996, 61, 4196.

(e) Maki, T.; Ishihara, K.; Yamamoto, H. Tetrahedron 2007, 63, 8645 .

(f) Arnold, K.; Davies, B.; Herault. D.; Whiting, A. Angew. Chem., Int. Ed. 2008, 47, 2673.

(g) Hosseini-Sarvari, M.; Sodagar, E.; Doroodmand, M. M. J. Org Chem. 2011, 76, 2853.

(h) Komura, K.; Nakano, Y.; Koketsu, M. Green Chem. 2011, 13, 828.

(i) Ojeda-Porras, A.; Gamba-Sánchez, D. J. Org. Chem. 2016, 81, 11548.

[8] (a) Beste, L. F.; Houtz, R. C. J. Polym. Sci., Part A: Polym. Chem. 1952, 8, 395.

(b) Miller, I. K. J. Polym. Sci., Polym. Chem. Ed. 1976, 14, 1403.

(c) McKinney, R. J. US 5302756, 1994.

(d) McKinney, R. J. US 5395974, 1995.

(e) Bon, E.; Bigg, D. C. H.; Bertrand, G. J. Org. Chem. 1994, 59, 4035 .

(f) Suggs, J. W.; Pires, R. M. Tetrahedron Lett. 1997, 38, 2227.

[9] (a) Stephenson, N. A.; Zhu, J.; Gellman, S. H.; Stahl, S. S. J. Am. Chem. Soc. 2009, 131, 10003.

(b) Hoerter, J. M.; Otte, K. M.; Gellman, S. H.; Cui, Q.; Stahl, S. S. J. Am. Chem. Soc. 2008, 130, 647.

(c) Kissounko, D. A.; Hoerter, J. M.; Guzei, L. A.; Cui, Q.; Gellman, S. H.; Stahl, S. S. J. Am. Chem. Soc. 2007, 129, 1776.

(d) Hoerter, J. M.; Otte, K. M.; Gellman, S. H.; Stahl, S. S. J. Am. Chem. Soc. 2006, 128, 5177.

(e) Kissounko, D. A.; Guzei, L. A.; Gellman, S. H.; Stahl, S. S. Organometallics 2005, 24, 5208 .

(f) Eldred, S. E.; Stone, D. A.; Gellman, S. H.; Stahl, S. S. J. Am. Chem. Soc. 2003, 125, 3422.

[10] Dineen, T. A.; Zajac, M. A.; Myers, A. G. J. Am. Chem. Soc. 2006, 128,16406 .
[11] Zhang, M.; Imm, S.; Neubert, B. S. L.; Neumann, H.; Beller, M. Angew. Chem., Int. Ed. 2012, 51, 3905.

[12] Shi, M.; Cui, S. C. Synth. Commun. 2005, 35, 2847.

[13] Tamura, M.; Tonomura, T.; Shimizu, K.; Satsuma, A. Green Chem. 2012, 14, 717.

[14] Becerra-Figueroa, L.; Ojeda-Porras, A.; Gamba-Sánchez, D. J. Org. Chem. 2014, 79, 4544.

[15] Pathare, S. P.; Jain, A. K. H.; Akamanchi, K. G. RSC Adv. 2013, 3, 7697.

[16] Ali, M. A.; Siddiki, S. M. A. H.; Kon, K.; Shimizu, K.-I. Tetrahedron Lett. 2014, 55, 1316.

[17] Nguyen, T. B.; Sorres, J.; Tran, M. Q.; Ermolenko, L.; Al-Mourabit, A. Org. Lett. 2012, 14, 3202.

[18] Allen, C. L.; Atkinson, B. N.; Williams, J. M. J. Angew. Chem., Int. Ed. 2012, 51, 1383.

[19] Wu, J.-W.; Wu, Y.-D.; Dai, J.-J.; Xu, H.-J. Adv. Synth. Catal. 2014 $356,2429$.

[20] (a) Rao, S. N.; Mohan, D. C.; Adimurthy, S. Org. Lett. 2013, 15, 1496.

(b)Yang, X.; Fan, L. L.; Xue, Y. RSC Adv. 2014, 4, 30108

[21] Nguyen, T. B.; Ermolenko, L.; Dau, M. E. T. H.; Al-Mourabit, A. Heterocycles 2014, 88, 403.

[22] Nageswara Rao, S.; Chandra Mohan, D.; Adimurthy, S. Green Chem. 2014, 16, 4122.

[23] Dusselier, M.; Van Wouwe, P.; Dewaele, A.; Makshina, E.; Sels, B. F. Energy Environ. Sci. 2013, 6, 1415.

[24] Maki-Arvela, P.; Simakova, I. L.; Salmi, T.; Murzin, D. Y. Chem. Rev. 2014, 114, 1909

[25] Li, L.; Fan, E.; Guan, Y.; Zhang, X.; Xue, Q.; Wei, L.; Wu, F.; Chen, R. ACS Sustainable Chem. Eng. 2017, 5, 5224.

[26] Lan, K.; Xu, S.; Li, J.; Hu. C. ACS Omega 2019, 4, 10571.

[27] Han, X.; Li, L.; Wei, C.; Zhang, J.; Bao, J. J. Agric. Food Chem. 2019, 67, 7082 .

[28] Lamers, B. A. G.; Genabeek, B.; Hennissen, J.; de Waal, B. F. M.; Palmans, A. R. A.; Meijer, E. W. Macromolecules 2019, 52, 1200.

[29] Yang, J.; Tana, J.-N.; Gu, Y. Green Chem. 2012, 14, 3304.

[30] Fatahpour, M.; Hazeri, N.; Maghsoodlou, M. T.; Lashkari, M. J. Chin. Chem. Soc. 2017, 64, 1071.

[31] (a) Zhao, C. Y.; Liu, J. Y.; Wang, Y.; Zhao, X. J.; Yuan, B.; Yue, M. M. Synth. Commun. 2014, 44, 827.

(b) Yue, M. M.; Liu, J. Y.; Wang, Y.; Yuan, B. Chin. J. Org. Chem. 2014, 34, 190 (in Chinese).

(岳闽敏, 刘巨艳, 王英, 袁斌, 有机化学, 2014, 34, 190.)

(c) Zhang, L. J.; Liu, J. Y.; Wang, Y. Chin. J. Org. Chem. 2013, 33, 339 (in Chinese).

(张丽君, 刘巨艳, 王英, 有机化学, 2013, 33, 339.)

(d) Huang, H. J.; Liu, J. Y.; Ma, E. Z.; Cao, Y. Y. Chin. J. Org. Chem. 2015, 35, 2372 (in Chinese).

(黄海静, 刘巨艳，马恩忠，曹映玉，有机化学, 2015, 35, 2372.)

(e) Liu, J. Y.; Huang, H. J.; Jiao, D. Q. Chin. J. Org. Chem. 2017, 37, 1808 (in Chinese)

(刘巨艳, 黄海静, 焦德全, 有机化学, 2017, 37, 1808.)

(f) Fang, Z. X.; Liu, J. Y.; Qiao, Y. H. Chin. J. Org. Chem. 2018, 38, 1985 (in Chinese).

(房智兴, 刘巨艳, 乔艳红, 有机化学, 2018, 38, 1985.)

[32] (a) Fuentes de Arriba, A. L.; Seisdedos, D. G.; Simons, L.; Alcazar, V.; Raposo, C.; Moran, J. R. J. Org. Chem. 2010, 75, 8303.

(b) Thomas, M.; Clarhaut, J.; Tranoy-Opalinski, I.; Gesson, J. P.; Roche, J.; Rapot, S. Bioorg. Med. Chem. 2008, 16, 8109.

(c) Willis, M. C.; Snell, R. H.; Fletcher, A. J.; Woodward, R. L. Org. Lett. 2006, 8, 5089. 